\title{
Cosmological scalar field perturbations can grow
}

\author{
Miguel Alcubierre, ${ }^{1}$ Axel de la Macorra, ${ }^{2}$ Alberto Diez-Tejedor, ${ }^{3,4}$ and José M. Torres ${ }^{1}$ \\ ${ }^{1}$ Instituto de Ciencias Nucleares, Universidad Nacional Autónoma de México, \\ Circuito Exterior C.U., A.P. 70-543, México D.F. 04510, México \\ ${ }^{2}$ Instituto de Fisica, Universidad Nacional Autónoma de México, \\ Circuito Exterior C.U., A.P. 20-364, México D.F. 04510, México \\ ${ }^{3}$ Santa Cruz Institute for Particle Physics and Department of Physics, \\ University of California, Santa Cruz, CA, 95064, USA \\ ${ }^{4}$ Departamento de Física, División de Ciencias e Ingenierías, \\ Campus León, Universidad de Guanajuato, León 37150, México
}

(Dated: July 23, 2018)

\begin{abstract}
It has been argued that the small perturbations to the homogeneous and isotropic configurations of a canonical scalar field in an expanding universe do not grow. We show that this is not true in general, and clarify the root of the misunderstanding. We revisit a simple model in which the zero-mode of a free scalar field oscillates with high frequency around the minimum of the potential. Under this assumption the linear perturbations grow like those in the standard cold dark matter scenario, but with a Jeans length at the scale of the Compton wavelength of the scalar particle. Contrary to previous analyses in the literature our results do not rely on time-averages and/or fluid identifications, and instead we solve both analytically (in terms of a well-defined series expansion) and numerically the linearized Einstein-Klein-Gordon system. Also, we use gauge-invariant fields, which makes the physical analysis more transparent and simplifies the comparison with previous works carried out in different gauges. As a byproduct of this study we identify a time-dependent modulation of the different physical quantities associated to the background as well as the perturbations with potential observational consequences in dark matter models.
\end{abstract}

PACS numbers: 98.80.-k, 98.80.Jk, 95.35.+d, 03.50.-z

\section{INTRODUCTION}

It is difficult to overestimate the relevance of the Jeans instability in modern physical cosmology. In order to understand the emergence of cosmic structure, we need a mechanism that transforms the nearly homogeneous and isotropic early universe we infer from e.g. the cosmic microwave background observations, to the highly clumped one we can see today at the scale of galaxy clusters and below. In the standard cosmological scenario this transition is possible thanks to the instability of a scalar mode that appears when we couple dark matter (DM) to gravity, the gravitational Jeans instability.

In a universe dominated by a barotropic perfect fluid with equation of state $p=p(\varepsilon) \ll \varepsilon$, the behavior of the small perturbations in the energy density depends crucially on the speed of sound, $c_{s}^{2}=\partial p / \partial \varepsilon$, and the Hubble radius, $H^{-1}$ (throughout this paper we use natural units such that $c=\hbar=1$ ). Roughly speaking, we can distinguish three different regions in Fourier space [1]: $i$ ) On scales smaller than the Jeans length, $\lambda_{J}=c_{s}\left(\pi / G \varepsilon_{0}\right)^{1 / 2} \sim c_{s} H^{-1}$, the contrast in the energy density oscillates with damping amplitude, due to the stabilizing effect of pressure and the expansion of the universe, respectively. Here $G$ is the Newton constant and $\varepsilon_{0}$ is the background matter density. $i i)$ Above this length scale but still below the Hubble radius, self-gravity dominates and the contrast in the energy density grows: the Jeans instability comes into play. iii) Finally, at scales larger than the Hubble radius a relativistic understand- ing of the problem shows that the contrast in the energy density freezes. ${ }^{1}$

Perfect fluid structure formation then demands matter with a low speed of sound in order to have a window, $c_{s} / H<\lambda<1 / H$, where the Jeans mode is released and the perturbations can grow. Cold dark matter (CDM) represents the simplest realization of this scenario, for which one assumes $c_{s}^{2} \approx 0$ for all relevant modes. ${ }^{2}$

For a canonical scalar field we have $c_{s}^{2}=1$, see e.g. Ref. [3], and it is usually argued that this implies that small perturbations in the energy density do not grow [4]. This would seem to be a very serious argument against the whole scalar field DM program [5], so we find it mandatory to clarify the issue.

In this paper we will consider the simplest situation: a universe dominated by a real massive scalar field $\varphi$ satisfying the Klein-Gordon equation, $\left(\square+m^{2}\right) \varphi=0$.

\footnotetext{
1 Actually all these statements are gauge dependent. We can always choose to work in e.g. the uniform density gauge, where the contrast in the energy density vanishes identically at all scales, or in e.g. the synchronous gauge, where the contrast in the energy density grows even for those modes larger than the Hubble radius. It is only in terms of the conformal-Newtonian gauge that the behavior outlined in the previous paragraph makes sense, and it is only in this gauge that we can easily compare our results with those sketched in the three points above.

2 If DM consists of collisionless particles it is more appropriate to talk about a free-streaming, rather than a Jeans, length; however, the idea is similar, see e.g. Sections 10.2 and 10.3 in Ref. [2] for a discussion.
} 
$\underset{\lambda_{J}}{\stackrel{\text { damped oscillations }}{\mid} \text { growing mode } \underset{H^{-1}}{\mid} \text { freeze down }} \rightarrow \lambda$

FIG. 1: Behavior of the linear perturbations in the contrast to the energy density for different wavelengths $\lambda$. The Jeans length $\lambda_{J}$ depends on the matter content in the universe. For a perfect fluid $\lambda_{J} \sim c_{s} H^{-1}$, and structure formation demands $c_{s}^{2} \ll 1$. However, when a canonical scalar field oscillates with high frequency around the minimum of the potential we have $\lambda_{J} \sim m^{-1}$, even though $c_{s}^{2}=1$. As usual this picture should be understood in the conformal-Newtonian gauge.

Here the box is the d'Alembert operator in four spacetime dimensions, and $m$ is the mass of the scalar particle [this corresponds to a scalar field potential of the form $V(\varphi)=m^{2} \varphi^{2} / 2$, so that the mass is defined as $\left.m^{2} \equiv \partial^{2} V / \partial \varphi^{2}\right]$. The more interesting case with a complex field including the self-interactions and the presence of other matter components (e.g. radiation, baryons, etc) will be presented elsewhere. We will show that when the scalar field is slowly rolling down the potential, linear perturbations cannot grow, in accordance with common wisdom. However, when the scalar field oscillates with high frequency $m \gg H$ around the minimum of the potential term, the Jeans length is not given by the naive value $c_{s} H^{-1}$ one would guess from a perfect fluid analogy. Instead it is determined by the Compton wavelength of the scalar particle, $c_{s} m^{-1}$, and the evolution of perturbations larger than this scale almost mimics that of the standard CDM scenario even though $c_{s}^{2}=1$ (see Figure 1 for details). The main difference with respect to the standard CDM evolution (apart from the appearance of a nonvanishing Jeans length), is the presence of a timedependent modulation of the different physical quantities. We will present below both an analytical argument based on expansions of the solution of the relevant cosmological equations, and results from simple numerical simulations.

Similar results have been presented before in the literature, see for instance Refs. [6] (as far as we know the gravitational instability of a canonical scalar field -in the context of a static universe - was reported for the first time by Khlopov, Malomed and Zel'dovich in Ref. [7]). However, to our knowledge, we present for the first time a description of the problem in terms of gauge-invariant fields. Furthermore, note that our analysis does not rely on time averages and/or fluid identifications. Instead we present a well-defined series expansion that makes it possible to find analytic solutions order by order in the expansion parameter: it is well known that the time average of the product of two functions is not in general the product of the time averages of those functions, and also that a scalar field is not a perfect fluid 8], so one needs to take some care with the standard procedure. With this formalism the results emerge more naturally than in previous works, and it is convenient in order to identify the root of the misunderstanding; see Eq. (15) and the paragraphs below.
We will follow the notation in Chapters 7 and 8 of Ref. [1]; in particular we use the signature $(+,-,-,-)$ for the spacetime metric. We highly recommend this reference to the reader interested in the details about some of the definitions and conventions below. One should also mention that recently the problem of structure formation with a massive scalar field has been studied by performing full nonlinear numerical simulations of the EinsteinKlein-Gordon system, both in the relativistic [9] and the nonrelativistic 10 regimes. However, we believe that by studying the problem from the point of view of a mode analysis in perturbation theory one can more clearly separate the relevant physical mechanisms that come into play at different scales.

\section{THE HOMOGENEOUS AND ISOTROPIC BACKGROUND}

At very large scales the universe is (nearly) homogeneous and isotropic; that makes it possible to introduce the idea of a homogeneous and isotropic background. According to the current cosmological observations this background can be described in terms of a flat RobertsonWalker (RW) metric of the form

$$
d s^{2}=a^{2}\left(d \eta^{2}-d x^{2}-d y^{2}-d z^{2}\right) .
$$

Here $\eta$ is the conformal cosmological time, $(x, y, z)$ a spatial coordinate system comoving with the expansion, and $a(\eta)$ the scale factor. Conformal time $\eta$ is related to the standard comoving cosmological time $t$ through $t=\int a d \eta$. The expansion rate is codified in the Hubble parameter, $H=\mathcal{H} / a=a^{\prime} / a^{2}$, with the prime denoting the derivative with respect to conformal time. The spacetime symmetries in Eq. (11) guaranty that the background field cannot depend on the spatial coordinates, so that $\varphi(\eta, \vec{x})=\varphi_{0}(\eta)$. Under these assumptions the Klein-Gordon equation simplifies to

$$
\varphi_{0}^{\prime \prime}+2 \mathcal{H} \varphi_{0}^{\prime}+a^{2} m^{2} \varphi_{0}=0 .
$$

There are two different timescales in Eq. (2): on the one hand that associated to the cosmological expansion, $\mathcal{H}^{-1}=(a H)^{-1}$, and on the other that defined by the mass of the scalar field, $(a m)^{-1}$. In this paper we will concentrate on the case when the expansion rate of the universe is very slow when compared to the characteristic time of oscillation of the scalar field, that is $(a H)^{-1} \gg$ $(a m)^{-1}$, which implies $H \ll m$. This inequality is always satisfied at late times. We will briefly discuss the regime $H \gg m$ later on in this section.

In order to proceed we propose a solution of the form

$$
\begin{aligned}
\varphi_{0}(\eta) & =\varphi_{0}^{H}(\eta)\left[\varphi_{0}^{m}(\eta)+\mathcal{O}^{2}(H / m)\right], \\
\mathcal{H}(\eta) & =\mathcal{H}^{H}(\eta)[1+\mathcal{O}(H / m)] \\
a(\eta) & =a^{H}(\eta)\left[1+\mathcal{O}^{2}(H / m)\right] .
\end{aligned}
$$

Here functions with a superscript $H$ vary on cosmological timescales, $\left(f^{H}\right)^{\prime} \sim a H f^{H}$, whereas those 
with a superscript $m$ vary on timescales given by the mass of the scalar field, $\left(f^{m}\right)^{\prime} \sim a m f^{m}$. In principle, higher-order terms oscillate in time with high frequency. Consequently, the derivative of one of those terms is not necessarily suppressed in the series expansion, e.g. $d[\mathcal{O}(H / m)] / d \eta \sim a H$. That is the reason for which the series expansion of the Hubble parameter $\mathcal{H}$ has a linear term in $H / m$, even though such a term is not present in the expression for the scale factor $a$.

Introducing the ansatz (3) into the Klein-Gordon equation we obtain

$$
\varphi_{0}(\eta)=\frac{A M_{\mathrm{Pl}}}{(m \eta)^{3}}\left[\sin (m t)+\mathcal{O}^{2}(H / m)\right],
$$

with $A$ an integration constant, $M_{\mathrm{Pl}}=1 / \sqrt{8 \pi G}$ the reduced Planck mass, and where we have fixed an arbitrary phase to zero. For later convenience, and with no loss of generality, from now on we will also choose $A^{2}=24$. From the above expression we see that the scalar field oscillates around $\varphi_{0}=0$ with constant frequency $m$ in comoving time $t$ (so that the frequency will increase in conformal time $\eta$ as the universe expands), and an amplitude that decays as $1 / \eta^{3}$. Introducing this expression for $\varphi_{0}(\eta)$ into the Friedmann equations,

$$
\begin{aligned}
\mathcal{H}^{2} & =\frac{8 \pi G}{3} a^{2} \varepsilon_{0}, \\
\mathcal{H}^{\prime}-\mathcal{H}^{2} & =-4 \pi G a^{2}\left(\varepsilon_{0}+p_{0}\right),
\end{aligned}
$$

one finds

$$
\begin{aligned}
\mathcal{H}(\eta) & =\frac{2}{\eta}\left[1-\frac{3}{4}\left(\frac{H}{m}\right) \sin (2 m t)+\mathcal{O}^{2}(H / m)\right], \\
a(\eta) & =(m \eta)^{2}\left[1+\mathcal{O}^{2}(H / m)\right] .
\end{aligned}
$$

For completeness we can also integrate the expression for the comoving time to obtain

$$
m t=\int m a d \eta=\frac{(m \eta)^{3}}{3}\left[1+\mathcal{O}^{3}(H / m)\right] .
$$

In the above equations we have used the fact that the background energy density and pressure are given by

$$
\varepsilon_{0}=\frac{1}{2}\left(\frac{\varphi_{0}^{\prime 2}}{a^{2}}+m^{2} \varphi_{0}^{2}\right), \quad p_{0}=\frac{1}{2}\left(\frac{\varphi_{0}^{\prime 2}}{a^{2}}-m^{2} \varphi_{0}^{2}\right) .
$$

In particular we find that, to lowest order in the series expansion, the background energy density redshifts with the inverse of the comoving volume, $\varepsilon_{0} \sim 1 / a^{3}$, whereas for the background pressure we obtain $p_{0} \sim\left(1 / a^{3}\right) \cos (2 m t)$. Note that to this same order we cannot distinguish the expansion rate from that in a CDM universe, $\mathcal{H}=2 / \eta$, even tough $\left|p_{0}\right| \sim \varepsilon_{0}$ during the evolution. Interestingly, this does not depend on the mass of the scalar particle, as long as it is large enough when compared to the expansion rate of the universe.

Since $H / m \sim 2 /(m \eta)^{3}$, the condition $H \ll m$ demands $m \eta \gg 1$. In terms of the scalar field,

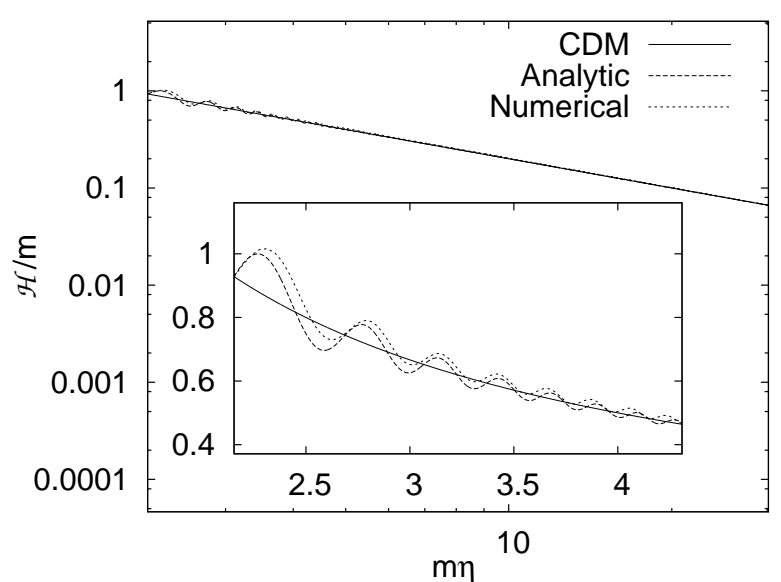

FIG. 2: Evolution of the conformal Hubble factor $\mathcal{H} / m$ as a function of the conformal time $m \eta$ for a free scalar field oscillating around the minimum of the potential. We show for comparison the solution in standard CDM (solid line), and both the analytical approximation in Eq. (6) (dashed line), and the full numerical solution of the Friedmann-KleinGordon system, Eqs. (2) and (5), (dotted line). In the figure the integration begins at $m \eta=10^{1 / 3}$, with $a=10^{2 / 3}$ and $\mathcal{H} / m=2 \times 10^{-1 / 3}$.

Eq. (4) above, we obtain $\left|\varphi_{0}\right| \ll M_{\mathrm{Pl}}$. That guarantees large values $\left|\epsilon_{\mathrm{sr}}\right|=\left|\eta_{\mathrm{sr}}\right|=2 M_{\mathrm{Pl}}^{2} / \varphi_{0}^{2} \gg 1$ of the slowroll parameters [11], with $\epsilon_{\mathrm{sr}} \equiv\left(M_{\mathrm{Pl}}^{2} / 2\right)\left(\partial_{\varphi} V / V\right)^{2}$ and $\eta_{\mathrm{sr}} \equiv M_{\mathrm{Pl}}^{2}\left(\partial_{\varphi}^{2} V / V\right)$, so we can safely conclude that the universe is not in a period of slow-roll inflation, as was evident from Eq. (6).

At this point one should mention that the dominant terms in the solution of the Friedmann-Klein-Gordon system given by Eqs. (4), (6) and (7) coincide with those of the exact solution found in Ref. [12] for a scalar field evolving in a universe dominated by a barotropic fluid such that $H=2 /(3 \gamma t)$, with $\gamma$ constant (when $\gamma=1$ this background barotropic fluid can be associated with the average energy density and pressure of the scalar field itself). Something similar happens with the solution reported in Ref. [13], obtained in terms of time averages. The main difference with our results is the fact that here we have made explicit the existence of higher-order terms in $H / m$, and in particular we have shown the first oscillating subdominant contribution to the Hubble parameter. Note however that this is not only a purely academic question: the inclusion of the higher-order terms will be crucial next for the understanding of the evolution of the small perturbations.

Figure 2 shows the evolution of the conformal Hubble factor $\mathcal{H} / m$ as a function of the conformal time $m \eta$ for a free scalar field oscillating around the minimum of the potential. The solid line corresponds to standard CDM, while the dashed and dotted lines show the case of the scalar field using both the analytical approximation in Eq. (6), and a full numerical solution of the Klein-Gordon and Friedmann system, Eqs. (2) and (5), respectively [for 
the numerical case we solve for $\mathcal{H}$ from Eq. (5b), and use Eq. (5a) to monitor the numerical error]. Notice that the evolution for the case of the scalar field follows closely that of CDM with small oscillations around it. Initially we have $H / m=0.2$ and the oscillations are still evident, but their amplitude decreases as time goes on.

Next we will consider the behavior of the small perturbations around the homogeneous and isotropic solution in Eqs. (4), (6), and (7). In particular, we find that an unstable Jeans mode grows at scales between the Compton length of the scalar particle and the Hubble radius of the universe; see Eq. (21) below for details.

\section{THE LINEAR PERTURBATIONS}

In this paper we will not consider vector and tensor modes, and will concentrate on the scalar sector of the perturbations. After all this is the sector that contains the Jeans mode of the theory. With this assumption, the most general expression for the spacetime metric of a universe close to a flat homogeneous and isotropic RW one takes the form

$$
\begin{aligned}
d s^{2} & =a^{2}\left\{(1+2 \phi) d \eta^{2}+2 \partial_{i} B d x^{i} d \eta\right. \\
& \left.-\left[(1-2 \psi) \delta_{i j}-2 \partial_{i} \partial_{j} E\right] d x^{i} d x^{j}\right\} .
\end{aligned}
$$

For the perturbed scalar field we will also write $\varphi=\varphi_{0}+\delta \varphi$. Here $\phi, B, \psi, E$ and $\delta \varphi$ are functions of the spacetime coordinates $\eta$ and $\vec{x}$, with $\delta \varphi \ll \varphi_{0}$ and $\phi, B, \psi, E \ll 1$.

All of these fields depend on the choice of coordinates used to write the line-element in Eq. (10), and they could in principle be describing fictitious inhomogeneities. For this reason it is sometimes convenient to work with gauge-invariant fields, such as [1]

$$
\begin{aligned}
\Phi & =\phi-(1 / a)\left[a\left(B-E^{\prime}\right)\right]^{\prime}, \\
\Psi & =\psi+\mathcal{H}\left(B-E^{\prime}\right), \\
\overline{\delta \varphi} & =\delta \varphi-\varphi_{0}^{\prime}\left(B-E^{\prime}\right) .
\end{aligned}
$$

Note that the above fields coincide with the amplitude of the metric and the scalar field perturbations in the conformal-Newtonian (also known in the literature as the longitudinal) coordinate system, for which $B=E=0$.

To linear order in the new field variables, the 00 and $0 i$ Einstein field equations, essentially the Hamiltonian and momentum constraints, take the following form:

$$
\begin{aligned}
& \Delta \Psi-3 \mathcal{H}\left(\Psi^{\prime}+\mathcal{H} \Phi\right)=4 \pi G a^{2} \overline{\delta \varepsilon} \\
& =4 \pi G\left[\varphi_{0}^{\prime} \overline{\delta \varphi}{ }^{\prime}+a^{2} m^{2} \varphi_{0} \overline{\delta \varphi}-\varphi_{0}^{\prime 2} \Phi\right], \\
& \Psi^{\prime}+\mathcal{H} \Phi=4 \pi G \varphi_{0}^{\prime} \overline{\delta \varphi}
\end{aligned}
$$

where $\Delta$ is the Laplace operator in flat space, and where $\overline{\delta \varepsilon}=\delta \varepsilon-\varepsilon_{0}^{\prime}\left(B-E^{\prime}\right)$ is the gauge-invariant density perturbation. For a scalar field, and to this order in the series expansion, there are no anisotropic stresses and the $i \neq j$ field equations fix $\Phi=\Psi$. (Note that so far we are using two different series expansions: one in the small perturbations of the spacetime metric and the scalar field, and the other in the expansion rate of the universe. We will soon introduce a new one in terms of the Jeans length.)

In order to move forward we find it convenient to define the new quantities

$$
v=a\left(\overline{\delta \varphi}+\frac{\varphi_{0}^{\prime}}{\mathcal{H}} \Psi\right), \quad z=\frac{a \varphi_{0}^{\prime}}{\mathcal{H}} .
$$

The gauge-invariant field $v(\eta, \vec{x})$ is usually known as the Mukhanov-Sasaki variable, and the function $z$ depends only on the spacetime background, $z=z(\eta)$. Note that, leaving the scale factor aside, the Mukhanov-Sasaki variable represents the scalar field perturbation evaluated in the spatially flat gauge $\psi=E=0$ [14]. Whenever the scalar field dominates the evolution of the universe, Eqs. (12a) and (12b) simplify to

$$
\begin{aligned}
\Delta\left(\frac{a^{2} \Psi}{\mathcal{H}}\right) & =4 \pi G z^{2}\left(\frac{v}{z}\right)^{\prime}, \\
\left(\frac{a^{2} \Psi}{\mathcal{H}}\right)^{\prime} & =4 \pi G z^{2}\left(\frac{v}{z}\right) .
\end{aligned}
$$

These two equations can be combined to obtain

$$
v^{\prime \prime}-c_{s}^{2} \Delta v-\frac{z^{\prime \prime}}{z} v=0,
$$

with $c_{s}^{2}=1$ analogous to a "speed of sound" for a canonical scalar field [3]. The main reason for this identification is the similarity of Eq. (15) with that obtained for a barotropic perfect fluid with sound speed $c_{s}$; see e.g. Eq. (7.65) in Ref. 1]. Notice that even though here we are only interested in the case of a scalar field with no self-interactions, so far the analysis of the perturbations is general and valid for an arbitrary potential.

Equation (15) above fixes a characteristic length scale given by $c_{s} a\left|z^{\prime \prime} / z\right|^{-1 / 2}$ (in this paper we will work with comoving wavenumbers $k$, but we will talk about physical wavelengths $\lambda$, with $\lambda=2 \pi a / k$ ). This length provides us with a ruler to discriminate between large and small scales in the scalar field perturbations. The correct estimation of this length scale for the different physical situations - and in particular the relative size it takes when compared to the Hubble radius - lies precisely at the heart of the usual misunderstanding.

As we will find soon, see Eqs. (20) and (21) below, the scale $c_{s} a\left|z^{\prime \prime} / z\right|^{-1 / 2}$ determines the Jeans length of the scalar field. However, this quantity is not always related to the naive guess $c_{s} H^{-1}$ one would expect from a perfect fluid analogy. In order to clarify this point, let us consider two representative cases. When the scalar field is rolling down to the minimum of the potential we have $\varphi_{0}^{\prime} \sim a H \varphi_{0}$, which implies $\left|z^{\prime \prime} / z\right| \sim(a H)^{2}$. This fixes the characteristic Jeans length to the Hubble radius. Shortwavelength perturbations (when compared to this length scale) oscillate in space and time as $v \sim \sin \left(c_{s} k \eta+\vec{k} \cdot \vec{x}\right)$, with $c_{s}^{2}=1$. The Jeans mode is then stabilized and the 
perfect fluid analogy seems possible, i.e. small perturbations to the homogeneous and isotropic solutions do not grow. Consider, for instance, the case of the inflaton during a slow-roll regime, or a quintessence field in the present universe, as particular realizations of this scenario.

However, when the zero-mode of the scalar field is oscillating with high frequency $m \gg H$ around the minimum of the potential, we have instead $\varphi_{0}^{\prime} \sim a m \varphi_{0}$, which results in $\left|z^{\prime \prime} / z\right| \sim(a m)^{2}$. The new length scale is well inside the Hubble radius, making possible the growth of perturbations with $a H<k<c_{s}^{-1}$ am (remember that for a canonical scalar field $c_{s}^{2}=1$ ). Now the naive estimation $c_{s} H^{-1}$ for the Jeans length has nothing to do with the correct one, $c_{s} m^{-1}$.

Let us consider in more detail this second scenario. Notice that, using the solutions in Eqs. (4), (6), and (7), the "mass" term in Eq. (15) takes the form

$$
\frac{z^{\prime \prime}}{z}=-a^{2} m^{2}\left[1+6\left(\frac{H}{m}\right) \sin (2 m t)+\mathcal{O}^{2}(H / m)\right] \text {. }
$$

As mentioned before, this is a purely background quantity. For practical reasons we will work with periodic boundary conditions over a box of comoving size $L$. We can always take the limit $L \rightarrow \infty$ at the end of the calculations. Under this assumption, the general solution to
Eq. (15) can be written in the form

$$
v(\eta, \vec{x})=\frac{1}{L^{3 / 2}} \sum_{\vec{k} \neq 0}\left[C_{\vec{k}} v_{\vec{k}}(\eta) e^{i \vec{k} \cdot \vec{x}}+c . c .\right]
$$

Here $C_{\vec{k}}$ are some dimensionless integration constants that label the different possible solutions, c.c. denotes complex conjugate, and the functions $v_{\vec{k}}(\eta)$ satisfy the equation

$$
v_{\vec{k}}^{\prime \prime}+\omega_{\vec{k}}^{2}(\eta) v_{\vec{k}}=0, \quad \text { with } \quad \omega_{\vec{k}}^{2}(\eta)=k^{2}-\frac{z^{\prime \prime}}{z}
$$

Note that the boundary conditions demand $k_{i}=2 \pi n_{i} / L$, with $n_{i}= \pm 1, \pm 2, \ldots$ and $i=1,2,3$. The zero-mode $k=0$ is already included in the description of the spacetime background, and for that reason it does not appear in Eq. (17).

According to the Eq. (18) the behavior of the modefunctions $v_{\vec{k}}(\eta)$ depends crucially on the relative value between the square of the wavenumber $k^{2}$, and the background function $z^{\prime \prime} / z$. For those modes smaller than the Compton wavelength, $k \gg a m$, we obtain (remember that in the fast expansion regime the Compton wavelength is always well inside the Hubble radius, and then $a m \gg a H)$

$$
v_{\vec{k}}(\eta)=\frac{1}{\sqrt{2 k}}\left(1+\mathcal{O}^{2}(H / m, a m / k)\right) \exp \left[-i\left(1+\mathcal{O}^{2}(H / m, a m / k)\right) k \eta\right]
$$

whereas for modes larger than this quantity, $k \ll a m$, we find

$$
\begin{aligned}
v_{\vec{k}}(\eta)= & \frac{A(k)}{\sqrt{2 m}}\left\{B(k) \bar{z}-\frac{1}{5} B(k) a\left(\frac{k}{a m}\right)\left(\frac{k}{a H}\right)\left[\sin (m t)-\frac{3}{2}\left(\frac{H}{m}\right) \cos ^{3}(m t)+\mathcal{O}^{2}(H / m)\right]\right. \\
& -i B^{-1}(k) \bar{z} \int \frac{d(m \eta)}{\bar{z}^{2}}+\frac{i}{7} B^{-1}(k) \frac{1}{a^{2}}\left(\frac{k}{a m}\right)\left(\frac{k}{a H}\right)\left[\cos (m t)-\frac{3}{2}\left(\frac{H}{m}\right) \sin ^{3}(m t)+\mathcal{O}^{2}(H / m)\right] \\
& \left.+\mathcal{O}^{2}(H / m, k / a m)\right\},
\end{aligned}
$$

(or $v_{\vec{k}}=v_{\vec{k}}^{\mathrm{r}}+i v_{\vec{k}}^{\mathrm{i}}$ if we prefer to think in terms of two real, linearly independent mode-functions $v_{\vec{k}}^{\mathrm{r}}$ and $v_{\vec{k}}^{\mathrm{i}}$.) Here we have defined $\bar{z}=z / \sqrt{6} M_{\mathrm{Pl}}$. Also, the functions $v_{\vec{k}}(\eta)$ have been normalized so that $v_{\vec{k}}\left(v_{\vec{k}}^{*}\right)^{\prime}-v_{\vec{k}}^{\prime}\left(v_{\vec{k}}^{*}\right)=i$, with the convention that in the asymptotic limit $H / m \rightarrow 0$, $k /($ am $) \rightarrow \infty$, we recover standard massless plane waves, i.e. $v_{\vec{k}}(\eta)=e^{-i k \eta} / \sqrt{2 k}$ in Eq. (19a). In the above expression $A(k)$ is a phase factor, $|A(k)|^{2}=1$, and $B(k)$ is a real number necessary to connect the two regimes in Eqs. (19a) and (19b) at $k \sim a m$. For our purposes in this paper we will not need to determine these quantities.

Notice that, while factors of $a H / k$ are small in
Eq. (19a), this is not necessarily true for factors of $k / a H$ in the case of Eq. (19b), where they can even dominate for modes inside the Hubble radius, i.e. those modes with $a H<k<a m$. This implies that, for modes larger than the Compton wavelength, the lowest nonvanishing contribution to the Mukhanov-Sasaki variable is not given by $\lim _{k \rightarrow 0} v_{\vec{k}}(\eta) \sim \bar{z}-i \bar{z} \int d(m \eta) / \bar{z}^{2}$, as one could have naively expected. Incidentally the extra terms will be crucial to determine the behavior of the modes relevant for structure formation. Since $a H \sim \eta^{-1}$ and $a m \sim \eta^{2}$, eventually all modes reach this regime. This completes our discussion of the Mukhanov-Sasaki variable.

However, the Mukhanov-Sasaki variable is only an aux- 
iliary field (remember that this quantity is related to the perturbation in the scalar field evaluated in the spatially flat gauge). In order to make contact with observations we need to move our attention, for instance, to the Newtonian potential $\Psi$, or to the contrast in the energy density, $\overline{\delta \varepsilon} / \varepsilon_{0}$. Introducing the expressions for the Mukhanov-Sasaki variable, Eqs. (19), into e.g. Eq. (14a), we obtain for the Newtonian potential ${ }^{4}$

$$
\Psi_{\vec{k}}(\eta)=\sqrt{\frac{3}{2}} \frac{1}{M_{\mathrm{Pl}}} \times \begin{cases}\frac{i}{\sqrt{2 k}}\left(\frac{a H}{k}\right) \frac{1}{a} \cos (m t) e^{-i k \eta}, & \text { if } k \gg a m, \\ \frac{A(k)}{\sqrt{2 m}}\left[\frac{1}{5} B(k)+i B^{-1}(k)\left(\frac{a H}{k}\right)\left(\frac{a m}{k}\right) \frac{1}{a^{3}}\right], & \text { if } k \ll a m .\end{cases}
$$

whereas from the Hamiltonian constraint (12a), we find for the contrast in the energy density ${ }^{5}$

$$
\left.\frac{\overline{\delta \varepsilon}}{\varepsilon_{0}}\right|_{\vec{k}}=\sqrt{\frac{2}{3}} \frac{1}{M_{\mathrm{Pl}}} \times \begin{cases}\frac{-i}{\sqrt{2 k}}\left(\frac{k}{a H}\right) \frac{1}{a} \cos (m t) e^{-i k \eta}, & \text { if } k \gg a m, \\ \frac{A(k)}{\sqrt{2 m}}\left[-\frac{1}{5} B(k)\left(\frac{k}{a H}\right)^{2}-i B^{-1}(k)\left(\frac{m}{H}\right) \frac{1}{a^{3}}\right], & \text { if } a H \ll k \ll a m, \\ \frac{A(k)}{\sqrt{2 m}}\left[-\frac{6}{5} B(k)+9 i B^{-1}(k)\left(\frac{a H}{k}\right)\left(\frac{a m}{k}\right) \frac{1}{a^{3}}\right] \cos ^{2}(m t), & \text { if } k \ll a H .\end{cases}
$$

Note that the two expressions above have been reported only to the lowest non-vanishing order in the series expansions. A pattern of small-amplitude, highfrequency oscillations are expected around the expressions in Eqs. (20) and (21), but this will be enough for the purposes of this paper.

For modes larger than the Compton wavelength, $k<a m$, the Newtonian potential mimics the behavior of standard CDM: one of the solutions remains constant, while the other decreases in time with $H / a \sim \eta^{-5}$; see for instance Eq. (7.53) in Ref. [1] for details. On the other hand, for modes smaller than the Compton wavelength, $k>a m$, the solution oscillates in time with a decreasing amplitude, $H \sim 1 / \eta^{3}$. This decay in the amplitude of the Newtonian potential is characteristic of a barotropic perfect fluid $p=p(\varepsilon) \ll \varepsilon$ with a nonvanishing Jeans scale, $c_{s}^{2} \neq 0$.

Something similar happens for the contrast in the energy density, where high-frequency modes with $k>a m$ oscillate in time with damped amplitude, $1 / a^{2} H \sim \eta^{-1}$. In contrast, there appear two different regimes for the modes larger than the Compton wavelength. Perturbations larger than the Compton length but still smaller than the Hubble radius, $a H<k<a m$, can grow in time as $1 /(a H)^{2} \sim \eta^{2}$, or decrease as $1 /\left(H a^{3}\right) \sim \eta^{-3}$,

${ }^{4}$ Here we have used the identity

$$
\Psi_{\vec{k}}(\eta)=-8 \pi G \frac{z^{2}}{a^{2}}\left[\frac{\mathcal{H}}{2 k^{2}}\left(\frac{v_{\vec{k}}}{z}\right)^{\prime}\right],
$$

that relates, in Fourier space, the Mukhanov-Sasaki variable to the Newtonian potential. which is again the same behavior as in the standard CDM scenario. On the other hand, those modes larger than the Hubble radius, $k<a H$, freeze with a timedependent modulation in $\cos ^{2}(m t)$. It is possible to trace back this oscillatory dependency to a background term, $\mathcal{H}^{\prime}-\mathcal{H}^{2} \sim \cos ^{2}(m t) / a$, that appears in the expression for the contrast in the energy density, see Eqs. (23) and (24) in footnote 5. These oscillations are therefore not expected to appear in the case of a complex scalar field for which $\mathcal{H}^{\prime}-\mathcal{H}^{2} \sim 1 / a$.

In particular, the growing mode in the energy density contrast $\overline{\delta \varepsilon} / \varepsilon_{0} \sim 1 /(a H)^{2} \sim \eta^{2} \sim t^{2 / 3}$ (i.e. $\overline{\delta \varepsilon} / \varepsilon_{0} \sim a$ to the lowest order in the expansion series) at those scales larger than the Compton wavelength but smaller than the Hubble radius, $1 / m<\lambda<1 / H$, is usually identified with structure formation in the universe (see for instance Eq. (7.56) in Ref. [1]). Note however that the (large) oscillations for the contrast in the energy density of modes larger than the Hubble radius are not present in the case of CDM.

At this point it is interesting to stress once again the

5 Here we have used the identity

$$
\left.\overline{\frac{\delta \varepsilon}{\varepsilon_{0}}}\right|_{\vec{k}}=8 \pi G \frac{z^{2}}{a^{2}}\left[\left(\frac{1}{3}+\frac{\mathcal{H}^{\prime}-\mathcal{H}^{2}}{k^{2}}\right) \frac{1}{\mathcal{H}}\left(\frac{v_{\vec{k}}}{z}\right)^{\prime}-\left(\frac{v_{\vec{k}}}{z}\right)\right],
$$

that relates, in Fourier space, the Mukhanov-Sasaki variable to the contrast in the energy density. Using the evolution for the background universe we can write

$$
\frac{1}{3}+\frac{\mathcal{H}^{\prime}-\mathcal{H}^{2}}{k^{2}}=\frac{1}{3}\left[1-9\left(\frac{a H}{k}\right)^{2} \cos ^{2}(m t)+\mathcal{O}(H / m)\right] .
$$




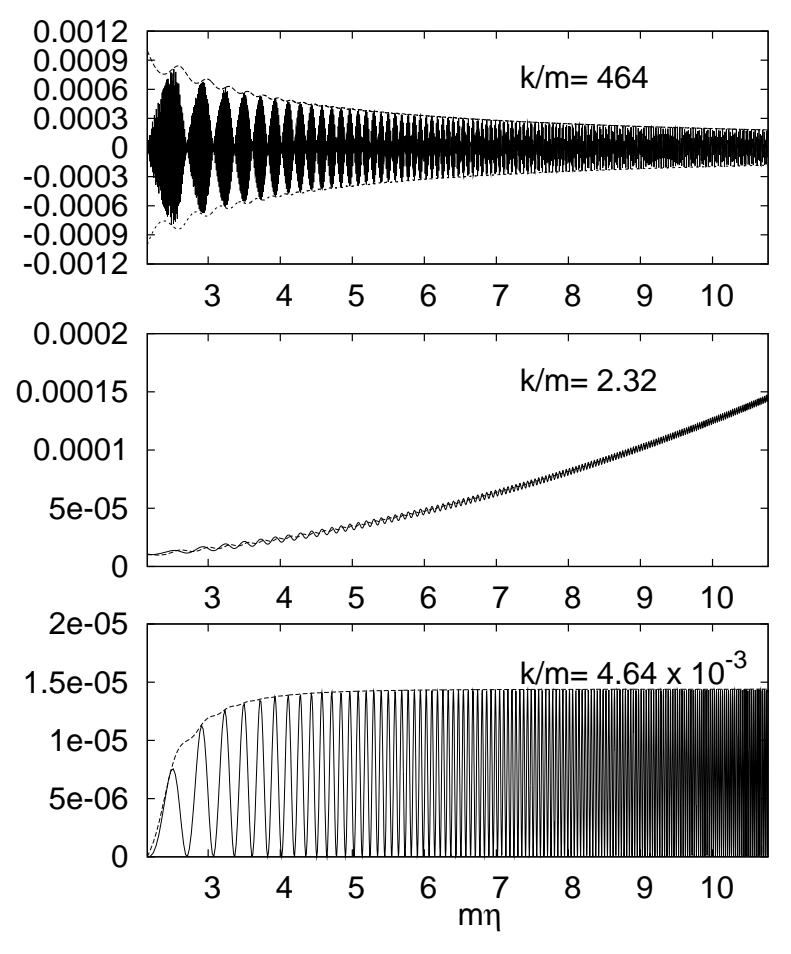

FIG. 3: Evolution of the contrast in the energy density, $\overline{\delta \varepsilon} /\left.\varepsilon_{0}\right|_{\vec{k}}$, as a function of conformal time $m \eta$ for three different Fourier modes, $k / m=\left(10^{2}, 1 / 2,10^{-3}\right) \times 10^{2 / 3}$, in the universe depicted in Figure 2 The lower and higher wavenumbers were chosen in order to lie outside of the region $[a H, a m]$ during the evolution. Solid lines represent the results of the numerical evolution, whereas dashed lines are obtained from an appropriate combination of the envelopes of the linearly independent solutions in Eq. (21). Modes shorter than the Compton wavelength (top panel) oscillate with damped amplitude. On the contrary, modes larger than the cosmological horizon (bottom panel) freeze, showing an oscillatory behavior associated to the inherent oscillation of the scalar field. Finally, those modes that are larger than the Compton wavelength but smaller than the Hubble radius (middle panel) grow at the same rate as the scale factor, as it is expected in the case of CDM. These modes can give rise to structures in the late universe. Note that since the equations are linear we can rescale the vertical axes in the figures arbitrarily, as long as the amplitudes remain always less than unity.

fact that these statements are only valid in the conformalNewtonian coordinate system, where the gauge-invariant fields $\Psi$ and $\overline{\delta \varepsilon} / \varepsilon_{0}$ take the same values as the Newtonian potential and contrast in the energy density, respectively. If we move to e.g. the synchronous coordinate system $\phi=B=0$, the contrast in the energy density is related to the gauge-invariant field $\overline{\delta \varepsilon} / \varepsilon_{0}$ through $\delta \varepsilon_{\mathrm{s}} / \varepsilon_{0}=\overline{\delta \varepsilon} / \varepsilon_{0}-\varepsilon_{0}^{\prime} /\left(a \varepsilon_{0}\right) \int a \Psi d \eta$, the constant of integration in this formula corresponding to an unphysical fictitious mode. As in the standard CDM scenario, the expression for intermediate wavelengths $a H \ll k \ll a m$ in Eq. (21) describes now all the modes larger than the Compton wavelength of the scalar particle, $k \ll a m$, and then in this gauge the contrast in the energy density grows with time as the scale factor, even for those modes that are well outside the Hubble horizon. (It is in this gauge that the matter power spectrum is usually presented in the literature.) Note that in this coordinate system the large oscillations in the energy density disappear, and then it is not clear for us if they could be observable in practice or if they are only a gauge artifact.

Figure 3 shows the evolution of the contrast in the energy density, $\overline{\delta \varepsilon} /\left.\varepsilon_{0}\right|_{\vec{k}}$, for three individual Fourier modes that are representative of the different regimes. For this figure we have considered the same background evolution of Figure 2, so that initially we have $m \eta=10^{1 / 3}$, $a=10^{2 / 3}$ and $\mathcal{H} / m=2 \times 10^{-1 / 3}$. In order to calculate the contrast in the energy density we integrate numerically Eqs. (14b) and (18) for the function $v_{\vec{k}}$ and the Newtonian potential $\Psi_{\vec{k}}$, simultaneously with the background evolution. We then use the Hamiltonian constraint (12a) to find the perturbation in the energy density, $\overline{\delta \varepsilon}_{\vec{k}}$.

The top panel in Figure 3 corresponds to a mode with $k / m=10^{8 / 3} \approx 464$, which is clearly in the regime $k>a m$ for the time interval $m \eta \in(2,11)$. We can see that the contrast in the energy density oscillates with an amplitude that decays like $1 /\left(a^{2} H\right)$, as expected. One can also clearly see from the figure that we have oscillations with two quite different frequencies: a high frequency oscillation coming from the term $e^{-i k \eta}$, modulated by a lower frequency oscillation that corresponds to the term $\cos (m t)$ in Eq. (21). The middle panel corresponds to a mode with $k / m=1 / 2 \times 10^{2 / 3} \approx 2.32$, so that $a H<k<a m$, also in the same time interval. In this case we see that the contrast in the energy density grows as $1 /\left(a^{2} H^{2}\right)$, with small oscillations. These modes are interesting for structure formation since they grow as if they were made of CDM. Finally, the bottom panel corresponds to a mode with $k / m=10^{-7 / 3} \approx 4.64 \times 10^{-3}$, for which we have $k<a H$ in the evolution. In this case the contrast in the energy density rapidly reaches a regime where it oscillates with constant amplitude. Notice that in all these three scenarios the frequency of the oscillations increases as time goes by. This is again to be expected since the frequency should be constant in cosmological time $t$, so that it increases in conformal time $\eta$.

\section{DISCUSSION}

For a perfect fluid with equation of state $p=p(\varepsilon) \ll \varepsilon$, there is a close relation between the Hubble radius and the Jeans length, $\lambda_{J} \sim c_{s} H^{-1}$. This is no longer true in the case of a canonical scalar field, where the characteristic Jeans length is fixed instead by $\lambda_{J} \sim c_{s} a\left|z^{\prime \prime} / z\right|^{-1 / 2}$. When this length scale is much smaller than the Hubble radius, perturbations can grow. For a perfect fluid this is possible only if $c_{s}^{2} \ll 1$. For a scalar field we have $c_{s}^{2}=1$, but we could still satisfy $a\left|z^{\prime \prime} / z\right|^{-1 / 2} \ll H^{-1}$. This is what happens, for instance, when the zero-mode of the scalar field is oscillating with high frequency (when 
compared to the expansion rate of the universe) around a minimum of the potential, where $\left.a\left|z^{\prime \prime}\right| z\right|^{-1 / 2} \sim m^{-1}$. Note that this value for the Jeans length at the scale of the Compton wavelength of the scalar particle cannot be resolved in terms of a nonrelativistic analysis, as it was previously done by $\mathrm{Hu}$ et al. in Ref. [5]. Indeed, the value of the Jeans length we identify in this paper does not coincide with the naive estimation they reported in Eq. (4) of that reference, and which has been frequently used after that (see e.g. Ref. [10]).

For those modes larger than the Jeans length the scalar field follows the evolution in the standard CDM scenario, except for the large oscillations of the contrast in the energy density for modes larger than the Hubble radius when evaluated in e.g. the conformal-Newtonian gauge. Note however that these (large) oscillations are not present in the behavior of the Newtonian potential, for which the scalar field behaves like CDM, or even in the super-Hubble modes of the contrast to the energy density when evaluated in e.g. the synchronous gauge. As far as we know these oscillations of the contrast in the energy density for long wavelength modes in certain coordinate systems had not been previously reported in the literature, and it will be very interesting to explore if they are only a gauge artifact or if on the contrary they could have imprinted some signatures on the cosmological observables. We expect these oscillations will not affect the large scale structure of the universe. After all, the distribution of galaxies is only sensitive to the subhorizon modes, and these modes follow the standard CDM evolution. However, they could affect e.g. the cosmic microwave background photons at large angular scales, which traced super-Hubble scales for a long period of time during the cosmological evolution. We leave a more detailed analysis of this point for a future work.

For those modes smaller than the Jeans length the evolution cannot bring the small perturbations in the early universe to the nonlinear regime, and the inhomogeneities are erased. This will introduce a cutoff in the mass power spectrum for the distribution of galaxies in the universe. Something similar happens in warm DM scenarios [15].
If the mass of the scalar particle lies at the scale of $10 \mu \mathrm{eV}$ or above, as it is expected for the QCD axion [16], the Jeans length would be smaller than a centimeter, and the growth of cosmic structures would be probably indistinguishable to that in the standard CDM scenario, at least while in the linear regime (see Ref. [9] for the case when nonlinearities become important). However, if we consider ultralight scalar particles of masses as low as $10^{-22} \mathrm{eV}$ [5, 17], the Jeans length grows to the scale of parsecs. This could have observable physical consequences in cosmology [10, 18], alleviating, for instance, the missing satellite discrepancy [19].

In order to determine properly the new expression for the mass power spectrum we would need a more elaborate analysis that includes, on the one hand, a knowledge of the initial conditions for the scalar field after inflation, as well as the evolution of the perturbations during the radiation dominated era. We leave this study for a future paper.

Note added.-After the first version of this paper was submitted for publication we became aware of Ref. [20], where the linearized Einstein-Klein-Gordon system is considered in the context of cosmological reheating. Although there are some similarities in the analysis, the motivation of our paper is different. We thanks Prof. James P. Zibin for pointing this reference out.

\section{Acknowledgments}

We are grateful to Juan Carlos Hidalgo, David Marsh and Darío Núñez for useful comments and discussions about a first version of this paper. This work was partially supported by PIFI, PROMEP, DAIP-UG, CAIPUG, the "Instituto Avanzado de Cosmología" (IAC) collaboration, DGAPA-UNAM under Grants No. IN115311 and No. IN103514, CONACyT México under grants No. 182445 and No. 167335, and PAPIIT-IN101415. ADT is also supported in part by Grant No. FQXi-1301 from the Foundational Questions Institute (FQXi).
[1] V.F. Mukhanov, Physical Foundations of Cosmology, Cambridge University Press (December 5, 2005) 421pp

[2] P. Coles and F. Lucchin, Cosmology, Wiley, 2 edition (June 15, 2002) 520pp

[3] J. Garriga and V.F. Mukhanov, "Perturbations in k-inflation," Phys. Lett. B 458 219-225 (1999) hep-th/9904176

[4] R.J. Scherrer, "Purely kinetic k-essence as unified dark matter," Phys. Rev. Lett. 93011301 (2004) astro-ph/0402316

[5] M. Dine and W. Fischler, "The not so harmless axion," Phys. Lett. B 120 137-141 (1983); E. kolb and M. Turner, The Early Universe, Chapter 10, Westview Press (February 21, 1994) 592pp; P. Sikivie, "Axion cosmology," Lect. Notes Phys. 741 19-50 (2008) astro-ph/0610440; A. Surez, V. Robles and T. Matos, "A review on the scalar field/Bose-Einstein condensate dark matter model," (2013) arXiv:1302.0903.

[6] B. Ratra, "Expressions for linearized perturbations in a massive scalar field dominated cosmological model," Phys. Rev. D 44 352-364 (1991); J. Hwang, "Roles of a coherent scalar field on the evolution of cosmic structures," Phys. Let. B 401 241-246 (1997) astro-ph/9610042; W. Hu, "Structure formation with generalized dark matter," Astrophys. J. 506 485-494 (1998); W. Hu, R. Barkana and A. Gruzinov, "Cold and fuzzy dark matter," Phys. Rev. Lett. 85 1158-1161 (2000) astro-ph/0003365; ; T. Matos and L.A. Ureña-Lopez, "A further analysis of a cosmological model of quintessence and scalar dark matter," Phys. Rev. D 63063506 
(2001) astro-ph/0006024; D.J.E. Marsh and P. Ferreira, "Ultra-light scalar fields and the growth of structure in the universe," Phys. Rev. D 82103528 (2010) [1009.3501]; C.G. Park, J.C. Hwang and H. Noh, "Axion as a cold dark matter candidate: Low-mass case," Phys. Rev. D 86083535 (2012) arXiv:1207.3124; A.J. Christopherson, J.C. Hidalgo and K.A. Malik, "Modelling non-dust fluids in cosmology," JCAP 01002 (2013) arXiv:1207.1870; L.A. Ureña-Lopez, "Non-relativistic approach for cosmological scalar field dark matter," Phys. Rev. D 90027306 (2014) arXiv:1310.8601.

[7] M.I. Khlopov, B.A. Malomed, and I.B. Zel'dovich, "Gravitational instability of scalar fields and formation of primordial black holes," MNRAS 215 575-589 (1985)

[8] A. Diez-Tejedor, "Note on scalars, perfect fluids, constrained field theories, and all that," Phys. Lett. B 727 27-30 (2013) arXiv:1309.4756

[9] J.M. Torres, M. Alcubierre, A. Diez-Tejedor and D. Nunez, "Cosmological nonlinear structure formation in full general relativity," Phys. Rev. D 90123002 (2014) arXiv:1409.7953

[10] H.Y. Schive, T. Chiueh and T. Broadhurst, "Cosmic structure as the quantum interference of a coherent dark wave," Nature Physics 10496499 (2014) arXiv:1406.6586]; H.Y. Schive, M.H. Liao, T.P. Woo, S.K. Wong, T. Chiueh, T. Broadhurst and W.Y.P. Hwang, "Understanding the core-halo relation of quantum wave dark matter, $\psi \mathrm{DM}$, from $3 \mathrm{D}$ simulations," (2014) arXiv:1407.7762

[11] A.R. Liddle and D.H. Lyth, Cosmological Inflation and
Large-Scale Structure, Cambridge University Press (April 13, 2000) 416pp

[12] A. de la Macorra and G. Piccinelli, "General scalar fields as quintessence," Phys. Rev. D 61123503 (2000)

[13] M.S. Turner, "Coherent scalar field oscillations in an expanding universe," Phys. Rev. D 281243 (1983)

[14] K.A. Malik and D. Wands, "Cosmological perturbations," Phys. Rept. 475 1-51 (2009) arXiv:0809.4944.

[15] P. Bode, J.P. Ostriker and N. Turok, "Halo formation in warm dark matter models," Astrophys. J. 556 93-107 (2001) astro-ph/0010389

[16] L. Visinelli and P. Gondolo, "Axion cold dark matter in view of BICEP2 results," Phys. Rev. Lett. 113011802 (2014) arXiv:1403.4594

[17] A. Arvanitaki, S. Dimopoulos, S. Dubovsky, N. Kaloper and J. March-Russell, "String axiverse," Phys. Rev. D 81123530 (2010) arXiv:0905.4720

[18] D.J.E. Marsh and J. Silk, "A model for halo formation with axion mixed dark matter," MNRAS 437 2652 (2013) arXiv:1307.1705; R. Hlozek, D. Grin, D.J.E. Marsh and P.G. Ferreira, "A search for ultra-light axions using precision cosmological data," Phys. Rev. D 91103512 (2015) arXiv:1410.2896

[19] A. Klypin, A.V. Kravtsov, O. Valenzuela and F. Prada, "Where are the missing galactic satellites?," Astrophys. J. 52282 (1999) arXiv:astro-ph/9901240

[20] R. Easther, R. Flauger and J.B. Gilmore, JCAP 04027 (2011) arXiv:1003.3011 